\title{
Characteristics of Vapor-Compression Cooling System on Multicomponent Working Medium
}

\author{
${ }^{\mathbf{1} A n n a}$ V. Pleshka, ${ }^{2}$ Arthur R. Valinurov \\ ${ }^{1,2}$ Naberezhnye Chelny Institute, Kazan Federal University, Naberezhnye Chelny, Republic of Tatarstan, Russian \\ Federation \\ Email: anya.pleshka@mail.ru
}

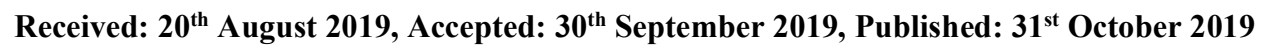

\begin{abstract}
The article presents the structural diagram of the static mathematical model for vapor compression cooling system (VC CS), which is the sequence of units with known transfer functions of thermal processes and the static mathematical model that allows to calculate the static characteristics of the considered CS in the form of cooling capacity dependence on the ambient temperature and the mass flow rate of cooling water.

The non-azeotropic mixture R423A was chosen as the working agent for static characteristics development, as the most environmentally safe among single-component refrigerants, and with greater energy efficiency for reverse cycles of heat engines. The evaluation criterion was the condition of lower energy consumption for a working agent compression in the compressor at a sufficiently high value of the refrigeration coefficient.

The calculation result showed that with the cooling water temperature decrease at the inlet to the condenser $t_{w 1}$ with a constant flow of cooling water $\mathrm{G}_{\mathrm{w}}$, the heat load of the evaporator $\mathrm{Q}_{\mathrm{ev}}$ will increase. A sharp increase of the evaporator heat load $Q_{e v}$ was also observed with flow rate increase $G_{w}$ to $0.4 \mathrm{~kg} / \mathrm{s}$, and with a further increase of cooling water flow rate, the heat load of the evaporator gradually increases. The dependence of the heat load of the evaporator $\mathrm{Q}_{\mathrm{ev}}$ on the temperature of the cooling water $\mathrm{t}_{\mathrm{w} 1}$ is linear.
\end{abstract}

Keywords

Steam Compression Cooling System, Static Mathematical Model, Freon, Compressor, Boiling, Evaporation, Temperature Head.

\section{Introduction}

Steam compression chillers are the most common for artificial cooling in the moderate cold area [1]. They are widely used in cryogenic and refrigeration equipment for cooling, freezing and storage due to their energy efficiency (lower energy consumption as compared to other machines) and less environmental hazard. Currently, refrigeration equipment does not have one-component working substances with simultaneously high thermodynamic, thermophysical and environmental indicators, and in this regard, they started to apply mixtures consisting of onecomponent substances authorized for production and use by the Montreal Protocol [2].

In particular, non-azeotropic refrigerants have such advantages that during the circulation of the working fluid along the circuit of the refrigeration system, its composition changes, which can lead to cooling capacity and refrigeration coefficient increase as compared with the characteristics for pure refrigerants [3]. Thus, changing the composition of non-azeotropic mixtures over a wide range, it is possible to obtain such properties of the working fluid that will provide the greatest (in specific conditions) efficiency of the refrigeration machine, which is estimated by the refrigeration coefficient. When they use non-azeotropic refrigerants, the aim is to increase refrigeration capacity, reduce the temperature of the end of compression, improve the circulation conditions of oil in the system, expand the range of applications for boiling and condensation temperatures, and provide the desired combination of refrigeration capacity with pressure levels. In some cases, the use of non-azeotropic mixtures can reduce the influence of the finite temperature difference on the efficiency of the thermodynamic cycle by performing cycles with variable boiling and condensation temperatures. At that, during vapor compression cooling system design operating on these mixtures, it is necessary to take into account such features as evaporation with increasing temperature, condensation with decreasing temperature, the influence of mixture component concentration on heat transfer coefficients, and other factors.

Besides, in the absence of control actions, the system can switch to a new operating mode with a possible deterioration in performance, which necessitates automatic control [4].

Therefore, the aim of this work is to develop a static mathematical model and calculate the static characteristics of a vapor compression cooling system on a multicomponent working agent.

\section{Methods}

Instead of refrigerants prohibited by the Montreal Protocol, it is proposed to use alternative refrigerants that comply with international environmental agreements [5]. Compression chillers use not only pure refrigerants and azeotropic mixtures, but also non-azeotropic mixtures, the distinguishing feature of which is the difference in the equilibrium concentrations of the components in the liquid and gas phases [6]. Changing the composition of non-azeotropic mixtures over a wide range, it is possible to obtain such properties of the working fluid that will provide the greatest 
(in specific conditions) efficiency of a refrigeration machine. Given this, initially one-component refrigerants were selected that are acceptable in terms of environmental performance, toxicity and flammability standards. Therefore, the calculations were performed for non-azeotropic mixtures containing these components. Further selection of refrigerants was made according to the main energy indicators of the cooling system, based on the conditions of energy cost reduction for the working agent compression in the compressor and the refrigeration coefficient increase. Energy performance was estimated using data interpolation from the tables of thermodynamic properties of multicomponent refrigerants [7]. During the analysis of the calculation results, the non-azeotropic refrigerant R423A was selected for further research.

The static mathematical model for the study was compiled according to the works [8,9], while the basis for its development was the structural diagram of the simulated object, which is the sequence of units with known static characteristics of elementary thermal processes (Fig. 1) [10]. For the model of the vapor compression cooling system under consideration, the determining influences are the mass flow rate of cooling water $G_{w}$ through the heat exchanger-condenser and its temperature $t_{w 1}$, and it is necessary to take into account the effect of the flow rate $G_{w}$ on the heat transfer coefficient of the heat exchanger-condenser $\mathrm{k}_{\mathrm{K}}$ tubes.

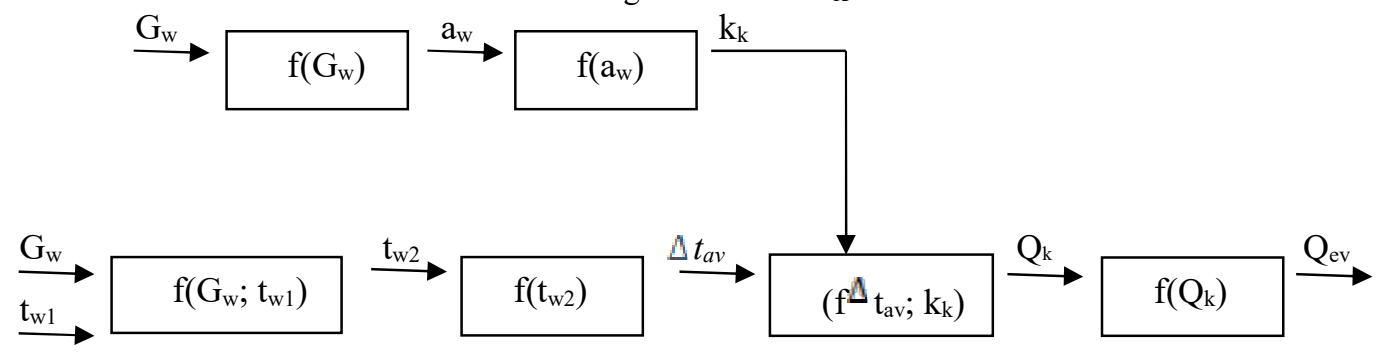

Figure 1: Block Diagram of the Vapor Compression Cooling System

In order to determine the first function $t_{\mathrm{w} 2}=f\left(G_{\mathrm{w}}, t_{w 1}\right)$ let's put down the heat balance equation for the heat exchanger-condenser, in which the heat from the hot working agent is removed by cooling water, while the working agent condenses and completely passes from the gaseous to the liquid state. The equation of the condenser heat balance has the following form, $\mathrm{kJ} / \mathrm{s}$ :

$\mathrm{Q}_{\mathrm{k}}=\mathrm{G}_{\mathrm{a}} \cdot \mathrm{q}_{\mathrm{k}}=\mathrm{G}_{\mathrm{w}} \cdot \mathrm{c}_{\mathrm{pw}} \cdot\left(\mathrm{t}_{\mathrm{w} 2}-\mathrm{t}_{\mathrm{w} 1}\right)$

where $\mathrm{Q}_{\mathrm{k}}$ is the thermal load on the capacitor, $\mathrm{kW} ; G_{\boldsymbol{a}}$ - mass flow rate of the working substance, $\mathrm{kg} / \mathrm{s} ; \mathrm{q}_{\mathrm{k}}=\mathrm{i}_{2}-\mathrm{i}_{3}-$ the specific heat consumption per unit flow rate of the refrigerant during condensation, $\mathrm{kJ} / \mathrm{kg}$, here $i_{2}$ and $i_{3}$ is the refrigerant enthalpy at the operating points of the cycle at the inlet and outlet of the heat exchanger-condenser; $t_{w 1}$ and $t_{w 2}$ - water temperature at the inlet and outlet of the heat exchanger-condenser, ${ }^{\circ} \mathrm{C} ; c_{p w}-$ specific mass isobaric heat capacity of water, $\mathrm{kJ} /(\mathrm{kg} \cdot \mathrm{K})$.

A stable thermal regime of the evaporator of the vapor compression cooling system during change of cooling water qualitative and quantitative parameters (temperature $t_{\mathrm{w} 1}$ and mass flow rate $G_{w}$ ) circulating through the heat exchanger-condenser can be achieved by excluding the water temperature tw 2 at the outlet of the condenser from the heat balance equation (1), based on the condition that it is not necessary to control its value, then we obtain the temperature of outlet water from the heat balance equation (1):

$\operatorname{tw} 2=\operatorname{tw} 1+\frac{G_{Q} \cdot q_{k}}{G_{w} \cdot c_{p u}}$

To determine the second function $\Delta t_{\mathrm{av}}=f\left(t_{w 2}\right)$ (the average temperature head in the heat exchanger-condenser) and simplify the mathematical transformation, we can use the arithmetic expression used for small values of the ratio $\Delta T_{\mathrm{b}} / \Delta T_{\mathrm{M}}<2$ of the coolant temperature differences circulating on both sides of the heat transfer surface, ${ }^{\circ} \mathrm{C}$ [6]. Considering the average temperature head as the arithmetic mean and taking into account the expression (2):

$$
\begin{aligned}
& \Delta \mathrm{t}_{\mathrm{av}}=\frac{\left(t_{k}^{J}-t_{w 1}\right)+\left(t_{k}-t_{w 2}\right)}{2}=\left[\frac{t_{k}^{J}}{2}-\frac{t_{w 1}}{2}+\frac{t_{k}}{2}-\frac{1}{2} \cdot\left(t_{w 1}+\frac{G_{a} \cdot q_{k}}{G_{w} \cdot c_{p w}}\right)\right]
\end{aligned}
$$

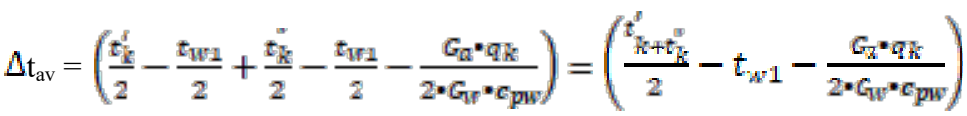

where $t_{\mathrm{K}}^{t}$ and $t_{\mathrm{K}}^{\prime \prime}$ - are the condensation temperatures of the refrigerant at the condensation pressure on the saturated liquid line and the saturated vapor line, respectively, ${ }^{\circ} \mathrm{C}$.

Given the known heat-transfer surface area $F_{K}$ of the heat exchanger-condenser tubes and the heat transfer coefficient $\mathrm{k}_{\mathrm{K}}$, in accordance with the Fourier law, we write the equation for the third function $Q_{\mathrm{K}}=f\left(\Delta t_{c p}\right)$ in the 
structural diagram (Fig. 1), which determines the value of the amount of heat transferred through the wall of the heat exchanger-condenser $\mathrm{kJ} / \mathrm{s}$ :

$\mathrm{Q}_{\mathrm{k}}=\mathrm{k}_{\mathrm{k}} \cdot \mathrm{F}_{\mathrm{k}} \cdot \Delta \mathrm{t}_{\mathrm{av}}$

where $\Delta \mathrm{t}_{\mathrm{av}}$ is the temperature head in the heat exchanger-condenser, ${ }^{\circ} \mathrm{C}$. The amount of heat transferred by the working agent to the cooling water through the wall of the heat exchanger-condenser in the process of its complete condensation is obtained by solving the system of three equations: the temperature of the cooling water at the outlet of the heat exchanger-condenser (2), the arithmetic mean temperature head (4) and heat transfer through the tube wall (5). This solution has the following form:

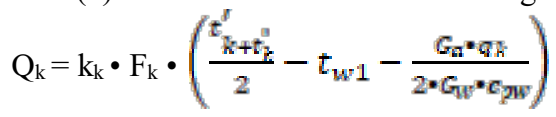

Heat transfer coefficient through the walls of the condenser tubes $k_{k}\left(G_{w}\right), W / m^{2 *} K$ :

$k_{k}\left(G_{W}\right)=\frac{1}{\frac{1}{\sigma_{W}\left(G_{W}\right)}+R_{T C k}+\frac{1}{\sigma_{k}}}$

Heat transfer coefficient from the water in the condenser $\alpha_{W}\left(G_{W}\right), \mathrm{W} / \mathrm{m} 2 * \mathrm{~K}$ :

$\mathrm{a}_{\mathrm{w}}\left(\mathrm{G}_{\mathrm{w}}\right)=\frac{N u_{w^{n}} \lambda_{\mathrm{w}}}{d_{\text {in }}}=\frac{\lambda_{\mathrm{w}}}{\mathrm{d}_{\text {in }}} \cdot\left(0,021 \cdot R e_{\mathrm{w}}^{0,8} \cdot P r_{\mathrm{w}}^{0,43} \cdot \varepsilon_{\mathrm{trns}}\right)$

where $N u_{w}$ is the Nusselt number; $\lambda_{w}$ - the coefficient of thermal conductivity, $\mathrm{W} /(\mathrm{m} \cdot \mathrm{K}) ; \mathrm{d}_{\text {in }}-$ the inner diameter of the heat exchanger-condenser tubes; $\operatorname{Re}_{w}$ - Reynolds number; $\operatorname{Pr}_{w}$ - Prandtl criterion; $E_{\text {trns }}=1-$ transition mode ratio.

$\mathrm{a}_{\mathrm{w}}\left(\mathrm{G}_{\mathrm{w}}\right)=\frac{\lambda_{\mathrm{W}}}{d_{\text {in }}} \cdot\left(0,021 \cdot\left(\frac{W^{*} \mathrm{Clin}_{i n}}{v_{\mathrm{W}}}\right) 0,8 \cdot P r_{\mathrm{w}}^{0,43} \cdot \mathrm{E}_{\text {trns }}\right)$

where $\mathrm{w}$ is the water velocity in the apparatus, $\mathrm{m} / \mathrm{s} ; v_{w}-$ kinematic viscosity of water, $\mathrm{m}^{2} / \mathrm{s}$.

$\mathrm{a}_{\mathrm{w}}\left(\mathrm{G}_{\mathrm{w}}\right)=\frac{\lambda_{\mathrm{w}}}{d_{\text {in }}} \cdot\left[0,021 \cdot\left[\left(\frac{4 \cdot G_{w}}{\pi \cdot p_{\mathrm{w}} \cdot d_{i n}^{\mathrm{T}} \cdot n_{1 x}}\right) \cdot \frac{d_{i n}}{v_{w}}\right]^{0,8} \cdot P r_{\mathrm{w}}^{0,43} \cdot \varepsilon_{t r n s}\right]$

where $\rho_{w}$ - water density, $\mathrm{kg} / \mathrm{m}^{3} ; n_{1 x}$ - the number of pipes in one stroke of the heat exchanger.

Let's put down the fourth equation for the function $Q_{\mathrm{ev}}=f\left(Q_{\mathrm{k}}\right)$ on the structural diagram (1), which determines the thermal load on the vapor compression cooling system, characterized by the amount of heat $Q_{\text {ev }}$ removed from the heat carrier in the heat exchanger-evaporator. To do this, we compose the equation of material balance, based on the equality of the mass flow of the working agent circulating in the cooling system. We get the following equality:

$\mathrm{Q}_{\mathrm{ev}}=\mathrm{Q}_{\mathrm{k}}=\mathrm{Q}_{\mathrm{kom}}$

where $\mathrm{Q}_{\mathrm{k}}=\mathrm{G}_{\mathrm{k}} * \mathrm{q}_{\mathrm{k}}$ is the thermal load on the capacitor; $\mathrm{Q}_{\mathrm{ev}}=\mathrm{G}_{\mathrm{ev}} * \mathrm{q}_{\mathrm{ev}}$ is the heat load on the evaporator, here $\mathrm{q}_{\mathrm{ev}}=\mathrm{i}_{1}$ $-\mathrm{i}_{4}$ - the specific heat consumption per unit flow rate of the refrigerant during evaporation, $\mathrm{kJ} / \mathrm{kg}$, here $i_{1}$ and $i_{4}$ are the enthalpies of the refrigerant at the operating points of the cycle at the outlet and inlet of the heat exchangerevaporator.

Then the heat load of the heat exchanger-evaporator is the following:

$\mathrm{Q}_{\mathrm{ev}}=\mathrm{G}_{\mathrm{ev}} \cdot \mathrm{q}_{\mathrm{ev}}=\mathrm{G}_{\mathrm{k}} \cdot \mathrm{q}_{\mathrm{ev}}=\frac{Q_{h}}{q_{k}} \cdot \mathrm{q}_{\mathrm{ev}}=\frac{\mathrm{q}_{e v}}{q_{i \mathrm{t}}} \cdot \mathrm{Q}_{\mathrm{k}}$

Thus, after the conversion, we finally obtain a static mathematical model of a vapor compression cooling system on a multicomponent working agent, which will be represented by the following equation:

$\mathrm{Q}_{\mathrm{ev}}\left(\mathrm{G}_{\mathrm{w}}, \mathrm{t}_{\mathrm{w} 1}\right)=\frac{q_{\mathrm{gv}}}{q_{k}} \cdot \mathrm{k}_{\mathrm{k}}\left(\mathrm{G}_{\mathrm{w}}\right) \cdot \mathrm{F}_{\mathrm{k}} \cdot\left(\frac{t_{\mathrm{k}}^{t}+t_{\mathrm{k}}}{2}-t_{w 1}-\frac{G_{\mathrm{a}} \cdot q_{k}}{2 \cdot G_{w} * C_{\mathrm{pw}}}\right)$

\section{Results and Discussion}

The model allows you to determine the amount of heat removed in the evaporator from the coolant, depending on changes of cooling water temperature $t_{w l}$ and the mass flow rate $G_{w}$ of cooling water (Figure 2, Figure 3 ). 


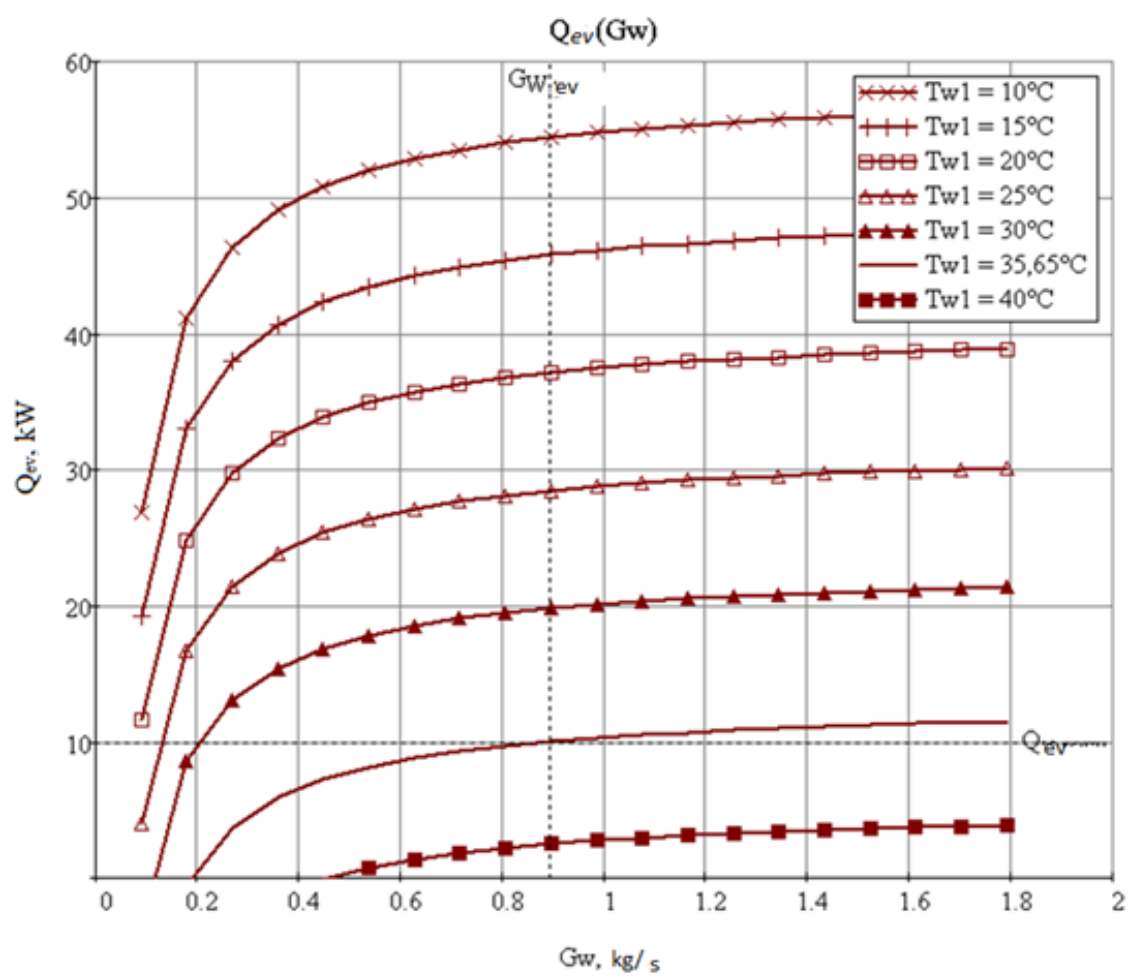

Figure 2: Dependence of the Evaporator Heat Load on the Flow of Cooling Water at Different Water Temperatures at the Inlet to the Condenser

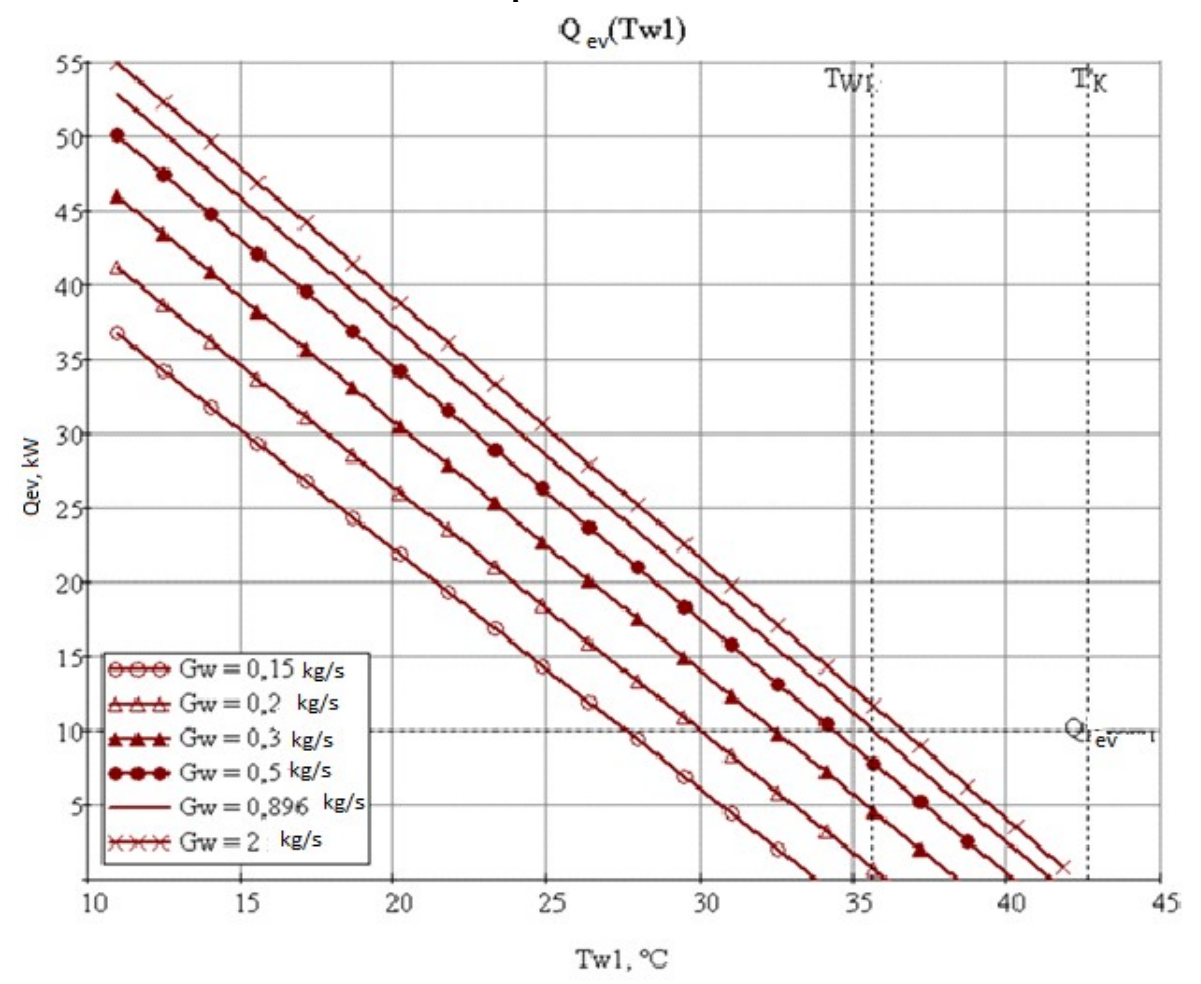

Figure 3: Dependence of the Evaporator Heat Load on the Temperature of Cooling Water at the Inlet to the Condenser at Different Flow Rates of Cooling Water

According to these graphs, it can be seen that with cooling water temperature decrease at the inlet to the condenser $t_{1}$ and its constant mass flow $G_{w}$, the heat load of the evaporator $Q_{и}$ will increase. Figure 2 shows a sharp increase of the evaporator heat load with the flow rate increase of cooling water $G_{w}$ to $0.4 \mathrm{~kg} / \mathrm{s}$. With a further increase of 
cooling water flow rate, the heat load of the evaporator increases smoothly. The dependence of the evaporator heat load on the temperature of the cooling water (Fig. 3) is linear.

\section{Conclusions}

Thus, the mathematical model was compiled and the static characteristics of the vapor compression refrigeration machine on a multicomponent working agent were calculated. They considered the dependence of the heat load of the evaporator on the flow of cooling water with a changing temperature of the water at the inlet to the condenser and the dependence of the heat load of the evaporator on the temperature of the cooling water at the inlet of the condenser with a changing flow of cooling water. The obtained static model makes it possible to evaluate the performance parameters of the steam compression cooling system under consideration: mass flow of the working agent, the amount of heat $\mathrm{Q}_{\mathrm{и}}$, the heat transfer coefficient in the condenser $\mathrm{k}_{\mathrm{K}}$ and the heat transfer surface area $\mathrm{F}_{\mathrm{K}}$ of the condenser.

\section{Acknowledgements}

The work is performed according to the Russian Government Program of Competitive Growth of Kazan Federal University.

\section{References}

1. Baranenko A.V. Refrigerators: the textbook for students of technical colleges studying "Engineering and Physics of Low Temperatures" / A.V. Baranenko, N.N. Bukharin, V.I. Pekarev, I.A. Sakun, L.S. Timofeevsky; Ed. by L.S. Timofeevsky. - St. Petersburg: Polytechnic, 1997. - 992 p.

2. Morozyuk T.V. Theory of refrigeration machines and heat pumps / T.V. Morozyuk. - Odessa: Studio "Negociant", 2006. - 712 p.

3. Babakin B.S. Alternative refrigerants and refrigeration system service based on them / B.S. Babakin, V.I. Stefanchuk, E.E. M.: Kolos, 2000. - 160 p.

4. Tatarenko Yu.V. Automation of the main elements of refrigeration machines: Textbook. / Yu.V. Tatarenko - St. Petersburg: ITMO University, 2015. - 43 p.

5. Tsvetkov, O.B., Laptev, Yu.A. Refrigerants and environment // Journal of Physics: Conference Series. 2017. Vol. 891. Article number 012218. pp. 1-3.

6. Mogorychny, V.I. Boiling of multicomponent working fluids used in refrigeration and cryogenic systems / V.I. Mogorychny, A.S. Dolzhikov // IOP Conf. Series: Journal of Physics: Conf. Series. 2017. Vol. 891. Article number 012022. pp. 1-13.

7. PC software: Reference Fluid Thermodynamic and Transport Properties v9. - Access mode: https://www.nist.gov/srd/refprop.

8. Karelin D.L., Gureev V.M., Muljukin V.L. Mathematical Model Of Liquid Vapor Compression System For Multicircuit Cooling Systems Of High Mobile Platform // International Journal of Applied Engineering Research. 2015. - №24. - pp. 45150-45155.

9. Karelin D.L., Boldyrev A.V., Boldyrev S.V., Belousov A.M. Modeling of dynamics of vapor compression cooling system // IOP Conference Series: Materials Science and Engineering. - 2018. - Vol.412, Is.1. - Art. № 012032. - pp. 1-4.

10. Ahmet K., Onder K., Ali K.Y. Performance and exergetic analysis of vapor compression refrigeration system with an internal heat exchanger using a hydrocarbon, isobutane (R600a). Int. J. Energy Res. 2008; 32:824-836. 